\title{
A NEW GLRT-BASED SHIP DETECTION TECHNIQUE IN SAR IMAGES
}

\author{
Pasquale Iervolino $^{\mathrm{a}}$, Raffaella Guida ${ }^{\mathrm{a}}$, Philip Whittaker ${ }^{\mathrm{b}}$ \\ ${ }^{a}$ Surrey Space Centre - University of Surrey Guildford, UK \\ \{P.Iervolino, R.Guida\}@surrey.ac.uk \\ ${ }^{\mathrm{b}}$ SSTL Ltd., Guildford, UK \\ P.Whittaker@sstl.co.uk
}

\begin{abstract}
This paper introduces a novel technique for ship-detection with Synthetic Aperture Radar (SAR) imagery based on the Generalized Likelihood Ratio Test (GLRT). Firstly, a suitable probability density function for a canonical ship is computed from the adoption of scattering models within the Geometrical Optic (GO) solution. Secondly, the GLRT is derived and the detector performance computed through Monte Carlo simulations. Finally, the GLRT technique is compared to the CFAR (Constant False Alarm Rate) algorithm in terms of ROC (Receiver Operating Characteristic) curves and computational load.
\end{abstract}

Index Terms - Synthetic Aperture Radar (SAR), scattering models, ship-detection, CFAR, GLRT.

\section{INTRODUCTION}

Synthetic Aperture Radars (SARs) are regarded as a useful mean to monitor the Earth activities thanks to their capability to acquire images independently from daylight and meteorological conditions and are considered an irreplaceable technology especially in security frameworks. One of these is the maritime surveillance, a topic of growing interest since the need for the maritime security, i.e. for monitoring the ocean pollution and tracking the movements of ships and cargos, is considerably increased during the last years.

In this scenario, ship-detection is one of the main applications. Traditional SAR ship-detection algorithms are mainly based on Constant False Alarm Rate (CFAR) methods: the sea background is characterized statistically and then the detector looks for individual pixels (or small group of pixels) whose brightness values are greater than a chosen threshold [1]. The main drawbacks of CFAR algorithms are that targets with intensity values very similar to those of sea clutter might not be detected and that the distribution parameters estimation needed to define the threshold is a computationally expensive procedure if nonGaussian models are adopted [2]. None of the algorithms already present in literature takes in account a model for the backscattering of the ship; as consequence, the detector may present a higher false alarm rate [1].

This paper introduces a novel model to consider the electromagnetic aspects behind the interactions of SAR signals with the ship and the surrounding sea by introducing an analytical closed form expression for the Radar Cross Section (RCS) backscattered from a canonical ship, adapted from a more generic model present in literature, in which some parameters are unknown and can only be described statistically. This is performed here and, once the statistical distribution of the ship RCS is also computed, a new detector based on the Generalized Likelihood Ratio Test (GLRT) is derived by evaluating the ratio of the ship likelihood function to the sea clutter likelihood function, as explained in the next sections.

The paper is organized as follows: in section 2 the scattering model is presented and the distribution function of the canonical ship is derived; in section 3 the novel GLRT is derived; in section 4 the simulations results are shown and GLRT and CFAR algorithms are compared; finally, in section 5 results are discussed and future perspectives briefly commented.

\section{SCATTERING FROM A CANONICAL SHIP}

In order to implement a GLRT, both the likelihood functions of the sea clutter and the ship must be defined. While the definition of a suitable distribution for the sea clutter is largely addressed in literature [1-4], the evaluation of the ship distribution has never been considered before. In [2] the authors modified the electromagnetic model in [5] by adapting it to the new scenario. First of all, some assumptions about the canonical shape and size of the ship are made to reduce the complexity of the problem: the ship is a perfect parallelepiped (hence, superimposed structures and tips are ignored), its hull is completely smooth, its dimensions are much larger than the working radar wavelength $\lambda$ and the sea clutter is modeled via a Gaussian stochastic process. In this scenario, both single and multiple scattering contributions should be analyzed; but, since the 
double reflection is the dominant mechanism, it will be the only contribution considered in the following. For the sake of completeness, the final formulation of the RCS is here reported for GO-GO approximation:

$$
\sigma=\frac{h\left|S_{p q}\right|^{2} l \tan \vartheta \cos \varphi\left(1+\tan ^{2} \vartheta \sin ^{2} \varphi\right) \exp \left[-\frac{\tan ^{2} \vartheta \sin ^{2} \varphi}{2 \sigma_{d e v}^{2}\left(2 / L^{2}\right)}\right]}{8 \pi^{2} \sigma_{d e v}^{2}\left(2 / L^{2}\right) \cos ^{2} \vartheta}
$$

In (1), $\sigma$ represents the RCS relevant to the double reflection contribution; $S_{p q}$ is the generic element of the scattering matrix with $p$ and $q$ standing for horizontal $\mathrm{H}$, or vertical, $\mathrm{V}$ polarization respectively; $l$ is the length of the portion of the ship belonging to the resolution cell, assuming the ship length larger than the SAR spatial resolution, $\sigma_{d e v}$ and $L$ are the standard deviation and the correlation length, respectively, of the stochastic process representing the sea clutter; $\varphi$ is the angle between the sensor line of flight and the projection of the ship hull onto the water surface; $\vartheta$ is the SAR look angle and $h$ is the height of the ship which contributes to form the dihedral surface between the sea and the ship hull (freeboard). In (1), $S_{p q}$ depends on the dielectric constant of the sea ( $\left.\varepsilon_{S W}\right)$, the dielectric constant of the hull $\left(\varepsilon_{H U L L}\right), \varphi, \vartheta$, the working wavelength $\lambda$ and the Fresnel coefficient according to the polarization of the propagating wave [5]. The parameters $\vartheta$ and $\lambda$ are a priori known and can be retrieved from the ancillary data of the SAR sensor; $\varepsilon_{S W}$ can be computed from the saline-water Double-Debye dielectric model presented in [6]; the ratio $\sigma_{\text {dev }} / L$ can be evaluated directly on the SAR image according to [7]. For the remaining ones $\left(\varepsilon_{H U L L}, \varphi\right.$ and $\left.h\right)$ suitable probability distribution functions need to be introduced bringing, in turn, to a probability density function for the RCS too [8].

In Table I, the distribution functions of the unknown parameters and the values of the a priori parameters are shown. All these parameters are used to compute the RCS of the canonical ship. In Fig.1 the probability density function (pdf) relative to the histogram and the fitting with a Gamma distribution are shown for $\mathrm{HH}$ polarization at $\mathrm{X}$ band $(9.65$ $\mathrm{GHz}$ ). The Gamma distribution passes the $\chi^{2}$ Goodness of Fit test $(p=52.54 \%)$ and can be used to model the backscattering return from the canonical ship.

In the next section, the GLRT detector is introduced and its way to operate explained.

\section{GLRT DETECTOR}

For the optimum detector, the target (the canonical ship in our case) needs to be accounted for in the statistical test.
Before deriving the statistical test, it is possible to define the test hypotheses:

$$
\begin{aligned}
& H_{0}: \text { sea clutter } \\
& H_{1}: \text { canonical ship }
\end{aligned}
$$

The test which maximizes the probability of detection (PD) for a given false alarm probability (optimum test) is the LRT (Likelihood Ratio Test), defined as follows [9]:

$$
\Lambda_{L}\left(\sigma_{x}\right)=\frac{p\left(\sigma_{x} / H_{1}\right)}{p\left(\sigma_{x} / H_{0}\right)}>\lambda(P F A) \leftrightarrow \text { Detected }
$$

where $\Lambda_{L}(\cdot)$ is the likelihood ratio function, $\sigma_{x}$ is the RCS of the pixel under test, $p\left(\sigma_{x} / H_{1}\right)$ and $p\left(\sigma_{x} / H_{0}\right)$ are the pdf of $\sigma_{x}$ given the target or the clutter is present respectively and $\lambda(P F A)$ is the threshold according to the desired probability of false alarm (PFA).

The LRT requires an explicit knowledge of the pdfs involved; however, in most of the real cases, the parameters of the pdfs are unknown and need to be estimated. When these parameters are estimated through maximum likelihood methods (MLE), the LRT becomes a GLRT defined as follows [9]:

$$
\Lambda_{G}\left(\sigma_{x}\right)=\frac{p\left(\sigma_{x} / H_{1}, \hat{\boldsymbol{\alpha}}_{t}\right)}{p\left(\sigma_{x} / H_{0}, \hat{\boldsymbol{\alpha}}_{c}\right)}>\lambda(P F A) \leftrightarrow \text { Detected }
$$

where $\Lambda_{G}(\cdot)$ is the generalized likelihood ratio function, $\hat{\boldsymbol{\alpha}}_{t}$ and $\hat{\boldsymbol{\alpha}}_{c}$ are the MLE estimators relative to the target (hypothesis $H_{1}$ ) and the clutter (hypothesis $H_{0}$ ) distribution parameters respectively. The GLRT is not the optimum statistical test, but if the pdfs of both clutter and target are well defined it can improve the Signal to Clutter Ratio (SCR). Indeed, $\Lambda_{G}<<1$ when $\sigma_{x}$ belongs to the clutter region, while $\Lambda_{G} \gg>1$ when $\sigma_{x}$ belongs to a potential target. Consequently, the output of the GLRT is an image where the clutter is attenuated and the targets are enhanced. The thresholding, therefore, can better operate and the performance of the GLRT is better compared with the standard CFAR algorithms. However, the GLRT needs to estimate the target parameters vector $\left(\hat{\boldsymbol{\alpha}}_{t}\right)$ and not only the clutter parameters vector $\left(\hat{\boldsymbol{\alpha}}_{c}\right)$ as CFAR algorithms do. As consequence, the GLRT presents a computational load higher than CFAR algorithms.

In the next section, the performance of the GLRT and CFAR are computed for a Gaussian distributed clutter (Exponentially-distributed intensity) and for a Gamma distributed target by letting the radar look angle and the roughness parameters ratio vary at $\mathrm{X}$ band $(9.65 \mathrm{GHz})$ and HH polarization. 
TABLE I: PARAMETERS NEEDED TO COMPUTE THE RCS OF THE SHIP

\begin{tabular}{|c|c|}
\hline Parameters & Value \\
\hline Radar look angle $\vartheta[\mathrm{deg}]$ & $40^{\circ}$ \\
\hline Radar wavelength $\lambda[\mathrm{m}]$ & 0.03 \\
\hline Roughness parameters $\sigma_{d e v} / L$ & 0.19 \\
\hline Length $l[\mathrm{~m}]$ & 3.3 \\
\hline Dielectric constant of the sea $\varepsilon_{S W}$ & $71.82-\mathrm{j} 37.78$ \\
\hline Dielectric constant of the ship hull & $\begin{array}{l}\text { Weighted mean of glass, } \\
\text { aluminum and steel } \\
\text { dielectric constants }\end{array}$ \\
\hline$\varepsilon_{H U L L}$ & $\sim U(0 ; 45)$ \\
\hline Orientation angle $\varphi[\mathrm{deg}]$ & $\sim U(0.2 ; 5.3)$ \\
\hline Freeboard height $h[\mathrm{~m}]$ &
\end{tabular}

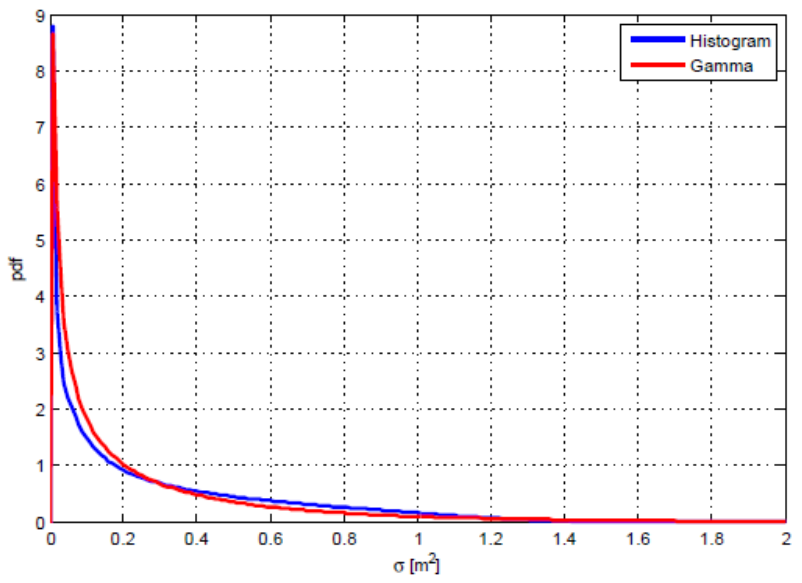

Fig. 1: Histogram of the RCS relevant to the double reflection contribution for $\mathrm{HH}$ polarization at $\mathrm{X}$ band and the Gamma distribution.

\section{MONTE CARLO SIMULATIONS}

In this section, the performance of the new GLRT technique is compared to that of the standard CFAR algorithm through simulations. Firstly, 200x200 pixels SAR images are simulated with a Monte Carlo method. As already anticipated in section 3, the clutter is Exponentiallydistributed with mean value set equal to the single scattering contribution from a rough surface within the GO approximation [5].

Secondly, all the pixels with normalized RCS lower than the typical NESZ (Noise Equivalent Signal Zero) of the TerraSAR-X sensor operating at $X$ band are set equal to the NESZ (NESZ=-25 dB [10]).

Thirdly, 100 values of RCS relative to the targets are simulated according to the Gamma distribution model presented in section 2 and shown in fig. 1 and are placed in random positions in the simulated SAR image.

Finally, each pixel is compared with the threshold and the PFA and PD values are computed.

In fig. 2, the ROC curves are retrieved after repeating the test (GLRT and CFAR) 100 times for each threshold. In
TABLE II: CFAR AND GLRT COMPUTATIONAL TIMES FOR ANALYZING 400000 PIXELS

\begin{tabular}{|c|c|c|}
\hline Operation & CFAR [s] & GLRT [s] \\
\hline Clutter parameters estimation & 0.296 & 0.296 \\
Target parameters estimation & n.a. & 1.385 \\
GLRT evaluation & n.a. & 0.193 \\
Thresholding & 0.361 & 0.361 \\
\hline TOT & 0.657 & 2.235 \\
\hline
\end{tabular}

general, it results that the performance of the novel GLRTbased technique is better (higher PD for any given PFA) than for the standard CFAR algorithm. Looking at the ROC curves on top of fig.2, where the radar look angle is fixed $\left(40^{\circ}\right)$ and the roughness ratio varies, it is shown that the performance gets worse when $\sigma_{d e v} / L$ increases because the radar backscattering from the sea clutter increases due to a rougher surface and, consequently, the SCR is reduced.

Looking at the ROC curves at the bottom of fig.2, instead, where $\sigma_{d e v} / L$ is fixed (0.2) and the radar look angle varies, it is highlighted that the performance gets better for an increase of the radar look angle because most of the incidence radiation of the sea clutter is reflected in the specular direction. However, for very high look angles $\left(9 \geq 50^{\circ}\right)$ also the RCS relevant to the target double reflection contribution diminishes and the SCR does not increase any more. As consequence, the performance of the detectors at $\vartheta=50^{\circ}$ is worse than the performance at $\vartheta=40^{\circ}$.

As already anticipated in the previous section, the computational load of the CFAR is lighter compared to the GLRT. In Table II, the computational times to derive a single $\operatorname{dot}\left(4 \cdot 10^{6}\right.$ pixels $)$ relative to the simulations shown in fig. 2 for both the CFAR and GLRT are reported. The computational load is evaluated using an Intel Pentium i52400 processor at $3.10 \mathrm{GHz}$. It results that the CFAR is more than 3 times faster than the GLRT because it does not need to estimate the target parameters.

\section{CONCLUSIONS}

A novel GLRT-based technique for ship-detection in SAR images has been presented and its performance evaluated against a traditional CFAR algorithm. Monte Carlo simulations show that GLRT performs better than CFAR for every ratio $\sigma_{d e v} / L$ and radar look angle $\vartheta$. The best results are obtained for $\sigma_{d e v} / L=0.2$ and $\vartheta=40^{\circ}$ for both approaches. Conversely, the CFAR results more than 3 times faster than the GLRT. However, both algorithms can be used in real time or near real time applications where the number of pixels to analyze is in the order of tens or hundreds millions. The authors are currently working to compute the simulated performance of both algorithms at different bands (S and C) and to perform the GLRT over real datasets retrieved from TerraSAR-X and Sentinel-1A sensors. 

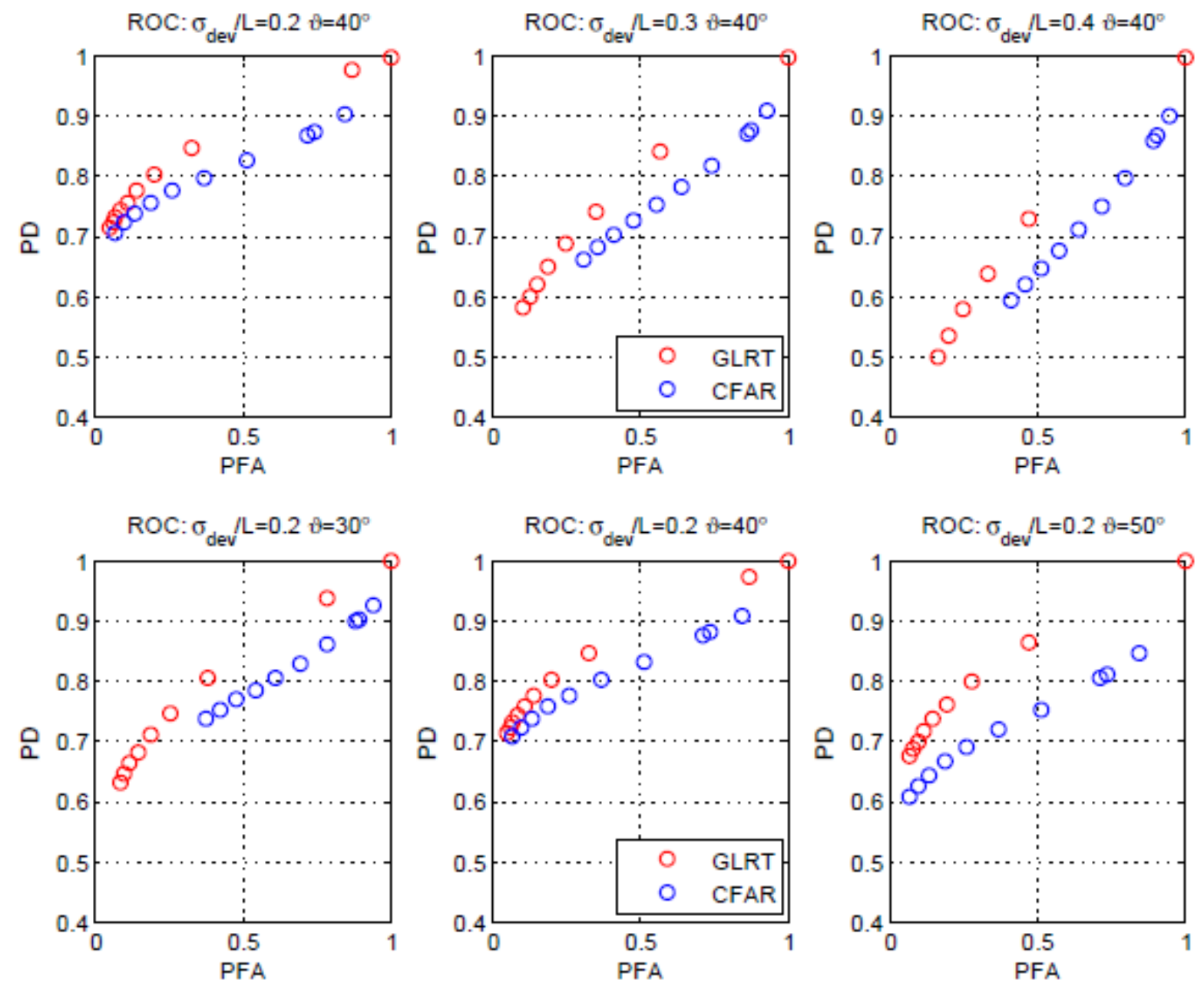

Fig. 2: ROC curves relative to the CFAR (blue dots) and GLRT (red dots) algorithms derived from SAR simulated images at $\mathrm{X}$ band and $\mathrm{HH}$ polarization for different values of the roughness ratio $\left(\sigma_{d e v} / L\right)$ and the radar look angle $(\vartheta)$.

\section{REFERENCES}

[1] D.J.Crisp, "The State-of-Art in Ship Detection in Synthetic Aperture Radar Imagery", DSTO Information Science Laboratory, 2004.

[2] P.Iervolino, R.Guida, and P.Whittaker, "NovaSAR-S and Maritime Surveillance”, Proc.IGARSS, pp. 1282-1285, 2013.

[3] X.Qin, S.Zhou, H.Zou, and G.Gao, "A CFAR detection algorithm for generalized gamma distributed background in HighResolution SAR images", IEEE Lett. Geosci. Remote Sens., vol. 10, no. 4, pp. 806-810, Apr. 2013.

[4] X.Xing, Z.L.Chen, H.Zou, and S.Zhou, "A fast algorithm based on two-stage CFAR for detecting ships in SAR images," Proc.APSAR, pp. 506-509, 2009.

[5] G.Franceschetti, A.Iodice, and D.Riccio, "A Canonical Problem in Electromagnetic Backscattering From Buildings",
IEEE Trans. on Geosci. Remote Sens., vol. 40, no.8, pp. 17871801, Aug. 2002.

[6] F.Ulaby, D.Long, Microwave Radar and Radiometric Remote Sensing, Ann Arbor, U Michigan, University of Michigan Press, 2013.

[7] P.Iervolino, R.Guida, and P.Whittaker, "Roughness Parameters Estimation of Sea Surface from SAR Images", Proc.IGARSS, pp. 5013-5016, 2014.

[8] P. Iervolino, R. Guida, P. Whittaker, "A model for the backscattering from a canonical ship in SAR imagery", IEEE J. Sel. Topics Appl. Earth Observ. Remote Sens., 2015, accepted

[9] S.Kay, Fundamentals of Statistical Signal Processing, Prentice Hall, vol. II,Upper Saddle River, NJ, USA, 2011.

[10] DLR: Radiometric Calibration of TerraSAR-X Data. Available at: http://www.astriumgeo.com/en/228-terrasarxtechnical-documents (Accessed: 21 May 2015). 